\title{
UNVEILING A NETWORK OF PARALLEL FILAMENTS IN THE INFRARED DARK CLOUD G14.225-0.506
}

\author{
Gemma Busquet $^{1,2}$, Qizhou Zhang ${ }^{3}$, Aina Palau ${ }^{4}$, Hauyu Baobab Liu ${ }^{5}$, Álvaro Sánchez-Monge ${ }^{6}$, Robert Estalella ${ }^{2}$, \\ Paul T. P. Ho ${ }^{3,5}$, Itziar de Gregorio-Monsalvo ${ }^{7,8}$, Thushara Pillai ${ }^{9}$, Friedrich Wyrowski ${ }^{10}$, \\ Josep M. Girart ${ }^{4}$, Fábio P. Santos ${ }^{11}$, and Gabriel A. P. Franco ${ }^{11}$ \\ ${ }^{1}$ INAF-Istituto di Astrofisica e Planetologia Spaziali, via Fosso del Cavaliere 100, I-00133 Roma, Italy; gemma.busquet@iaps.inaf.it \\ ${ }^{2}$ Departament d'Astronomia i Meteorologia, Institut de Ciències del Cosmos (ICC), Universitat de Barcelona (IEEC-UB), \\ Martí i Franquès 1, E-08028 Barcelona, Catalunya, Spain \\ ${ }^{3}$ Harvard-Smithsonian Center for Astrophysics, 60 Garden Street, Cambridge, MA 02138, USA \\ ${ }^{4}$ Institut de Ciències de l'Espai (CSIC-IEEC), Campus UAB, Facultat de Ciències, Torre C-5 parell, E-08193 Bellaterra, Catalunya, Spain \\ ${ }^{5}$ Academia Sinica Institute of Astronomy and Astrophysics, Taipei, Taiwan \\ ${ }^{6}$ INAF, Osservatorio Astrofisico di Arcetri, Largo E. Fermi 5, I-05125 Firenze, Italy \\ ${ }^{7}$ European Southern Observatory, Karl-Schwarzschild-Strasse 2, D-85748 Garching, Germany \\ 8 Join ALMA Observatory, Alonso de Córdova 3107, Vitacura, Santiago, Chile \\ ${ }^{9}$ Caltech Astronomy Department, MC 249-17, 1200 East California Boulevard, Pasadena, CA 91125, USA \\ ${ }^{10}$ Max-Planck-Institut für Radioastronomie, Auf dem Hügel 69, D-53121 Bonn, Germany \\ ${ }^{11}$ Departamento de Física-ICEx-UFMG, Caixa Postal 702, 30.123-970 Belo Horizonte-MG, Brazil \\ Received 2012 November 27; accepted 2012 December 23; published 2013 February 7
}

\begin{abstract}
We present the results of combined $\mathrm{NH}_{3}(1,1)$ and $(2,2)$ line emission observed with the Very Large Array and the Effelsberg $100 \mathrm{~m}$ telescope of the infrared dark cloud G14.225-0.506. The $\mathrm{NH}_{3}$ emission reveals a network of filaments constituting two hub-filament systems. Hubs are associated with gas of rotational temperature $T_{\text {rot }} \sim$ $15 \mathrm{~K}$, non-thermal velocity dispersion $\sigma_{\mathrm{NT}} \sim 1 \mathrm{~km} \mathrm{~s}^{-1}$, and exhibit signs of star formation, while filaments appear to be more quiescent $\left(T_{\text {rot }} \sim 11 \mathrm{~K}\right.$ and $\left.\sigma_{\mathrm{NT}} \sim 0.6 \mathrm{~km} \mathrm{~s}^{-1}\right)$. Filaments are parallel in projection and distributed mainly along two directions, at P.A. $\sim 10^{\circ}$ and $60^{\circ}$, and appear to be coherent in velocity. The averaged projected separation between adjacent filaments is between $0.5 \mathrm{pc}$ and $1 \mathrm{pc}$, and the mean width of filaments is $0.12 \mathrm{pc}$. Cores within filaments are separated by $\sim 0.33 \pm 0.09 \mathrm{pc}$, which is consistent with the predicted fragmentation of an isothermal gas cylinder due to the "sausage"-type instability. The network of parallel filaments observed in G14.225-0.506 is consistent with the gravitational instability of a thin gas layer threaded by magnetic fields. Overall, our data suggest that magnetic fields might play an important role in the alignment of filaments, and polarization measurements in the entire cloud would lend further support to this scenario.
\end{abstract}

Key words: ISM: clouds - ISM: individual objects (G14.225-0.506) - stars: formation

Online-only material: color figures

\section{INTRODUCTION}

Filaments are ubiquitous structures in star-forming complexes (e.g., Schneider \& Elmegreen 1979; Wiseman \& Ho 1998; Hatchell et al. 2005; Goldsmith et al. 2008; Wang et al. 2008; Jackson et al. 2010; Schneider et al. 2010; Molinari et al. 2010; André et al. 2010; Arzoumanian et al. 2011), and often intersect in high-density regions of low aspect ratio and associated with star formation, known as hub-filament systems (e.g., Myers 2009; Liu et al. 2012). However, their formation and their role in the star formation process are not yet well understood.

In nearby $(d \sim 200-500 \mathrm{pc})$ molecular clouds, recent photometric results from Herschel suggest that large-scale turbulence might be responsible for the formation of filaments (Arzoumanian et al. 2011), while spectroscopic studies, sensitive to smaller scales, show that filaments present subsonic nonthermal motions (Hacar \& Tafalla 2011; Pineda et al. 2011), indicative of a dissipation of turbulence at smaller scales. While this is consistent with observations in more distant and massive star-forming regions, such as G28.34+0.06 (Wang et al. 2008), a number of studies reveal supersonic non-thermal motions and suggest the formation of filaments by the convergence of flows or by filament-filament collisions on large scales (Schneider et al. 2010; Csengeri et al. 2011; Heitsch et al. 2008; Jiménez-Serra et al. 2010; Henshaw et al. 2013; Miettinen 2012; Nakamura et al. 2012). In addition, theoretical studies propose that magnetic fields could play a role in the formation of filaments (e.g., Nagai et al. 1998; Nakamura \& Li 2008). It is clear, then, that several formation mechanisms have been invoked to explain the formation and alignment of filaments and therefore further spectroscopic studies of filamentary regions are essential to investigate the origin and evolution of such structures.

Filaments are prevailing structures in infrared dark clouds (IRDCs; cf. Rathborne et al. 2006). In this Letter we present combined Very Large Array (VLA) and Effelsberg $100 \mathrm{~m}$ telescope observations of the $\mathrm{NH}_{3}(1,1)$ and $(2,2)$ transitions toward the IRDC G14.225-0.506 (hereafter G14.2). Most of the studies performed so far toward this region focus on the brightest infrared sources, IRAS 18153-1651 (hereafter I18153) and IRAS 18152-1658 (hereafter I18152) with a luminosity of $\sim 1.1 \times 10^{4} L_{\odot}$ and $\sim 4 \times 10^{3} L_{\odot}$, respectively, and located at a distance of $2.3 \mathrm{kpc}$ (Jaffe et al. 1981, 1982). Single-dish observations show that $\mathrm{I} 18153$ is associated with $\mathrm{H}_{2} \mathrm{O}$ maser emission (Jaffe et al. 1981; Palagi et al. 1993), and dense gas emission (Plume et al. 1992; Anglada et al. 1996; Bronfman et al. 1996). More recent VLA observations reveal $\mathrm{H}_{2} \mathrm{O}$ maser emission in nine different positions (Wang et al. 2006), which indicates that star formation is already ongoing in some parts of the cloud. IRDC G14.2 has been identified, using Spitzer data, by Peretto \& Fuller (2009) as a cloud containing an 


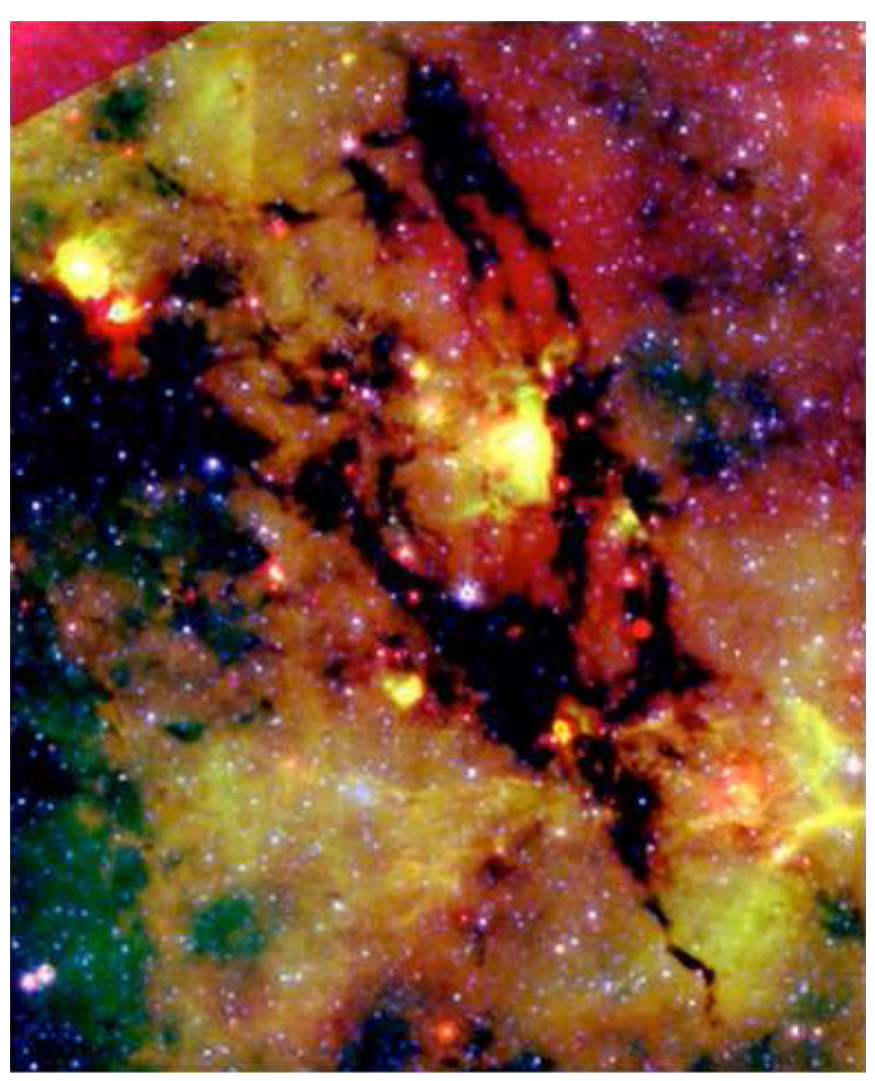

Figure 1. Archival Spitzer 4.5/8.0/24 $\mu \mathrm{m}$ (blue/green/red) three-color composite image of IRDC G14.225-0.506.

(A color version of this figure is available in the online journal.)

important amount $(\sim 100)$ of density enhancements or fragments displaying a filamentary morphology. The filamentary appearance of G14.2 (Figure 1) and its relatively nearby distance make this region a good selection to investigate the physical properties of filaments and their formation mechanism at high spatial resolution.

\section{OBSERVATIONS}

The observations of the $\mathrm{NH}_{3}(1,1)$ and $(2,2)$ transitions were conducted using the $\mathrm{VLA}^{12}$ in the D configuration on 2005 November 12 (project AW666). We performed a 34-pointing mosaic covering an area of $7^{\prime} \times 13^{\prime}$. The integration time was $\sim 4.5$ minutes per pointing. The adopted flux density of the flux calibrator 3C 286 was $2.41 \mathrm{Jy}$ at a wavelength of $1.3 \mathrm{~cm}$. The time variation of the gains was calibrated using J1832-105, with a bootstrapped flux of $0.97 \pm 0.01 \mathrm{Jy}$, and the bandpass calibrator used was 3C 273. We used the 4IF spectral line mode, which allows simultaneous observations of the $\mathrm{NH}_{3}(1,1)$ and $(2,2)$ lines with two polarizations for each line. The bandwidth used was $3.12 \mathrm{MHz}$, divided into 63 channels with a channel spacing of $48.8 \mathrm{kHz}\left(\sim 0.6 \mathrm{~km} \mathrm{~s}^{-1}\right.$ at $\left.1.3 \mathrm{~cm}\right)$, centered at $\sim 21 \mathrm{~km}$ $\mathrm{s}^{-1}$. The visibility data sets were calibrated using the AIPS software package of the NRAO.

To recover extended structures filtered out by the interferometer, we performed $\mathrm{NH}_{3}$ observations with the Effelsberg $100 \mathrm{~m}$ telescope (project 101-07). The observations were carried out

\footnotetext{
12 The VLA is operated by the National Radio Astronomy Observatory (NRAO). The NRAO is a facility of the National Science Foundation operated under cooperative agreement by Associated Universities, Inc.
}

between 2008 April 4 and 7. We used the 18-26 GHz HEMT receiver tuned to a frequency of $23.7 \mathrm{GHz}$ with the 16384 channel fast Fourier transform spectrometer, allowing simultaneous observations of the $\mathrm{NH}_{3}(1,1)$ and $(2,2)$ lines. The total bandwidth used was $100 \mathrm{MHz}$, which provides a velocity resolution of $0.075 \mathrm{~km} \mathrm{~s}^{-1}$. The observations were conducted in frequency switching mode with a frequency throw of $7.5 \mathrm{MHz}$. At the observed wavelength, the half-power beamwidth of the telescope is $\sim 40^{\prime \prime}$. The map covered an area of $8^{\prime} \times 13^{\prime}$ and was made by observing the positions of a grid with half-beam spacing. The pointing was checked at hourly intervals, with a pointing accuracy better than $8^{\prime \prime}$. To convert the arbitrary noise tube units of the Effelsberg data to main beam brightness temperature, we observed as a primary flux calibrator NGC 7027 and a nearby quasar as a secondary flux calibrator. Data reduction was performed using the CLASS package, which is part of the GILDAS $^{13}$ software. We combined the visibility data from the VLA and Effelsberg $100 \mathrm{~m}$ telescope for both $\mathrm{NH}_{3}(1,1)$ and $\mathrm{NH}_{3}(2,2)$ lines following the MIRIAD procedure outlined in Vogel et al. (1984). We applied a $u v$-taper function of $23 \mathrm{k} \lambda$ during imaging. The resulting synthesized beams were $8^{\prime \prime} .2 \times 7^{\prime \prime} .0$ (P.A. $=-15^{\circ}$ ) for $\mathrm{NH}_{3}(1,1)$ and $8^{\prime \prime} .0 \times 6^{\prime \prime} .9$ (P.A. $=-16^{\circ}$ ) for $\mathrm{NH}_{3}(2,2)$. The rms was $\sim 8 \mathrm{mJy}^{\text {beam }}{ }^{-1}$ per $0.6 \mathrm{~km} \mathrm{~s}^{-1}$ spectral channel.

\section{RESULTS AND ANALYSIS}

Figure 2 (top left) shows the combined (VLA+Effelsberg) zero-order moment map of the $\mathrm{NH}_{3}(1,1)$ emission overlaid on the $8 \mu \mathrm{m}$ Spitzer image. The overall morphology of the $\mathrm{NH}_{3}(1,1)$ dense gas consists of extended and clumpy filamentary structures, strikingly mimicking the extinction feature seen in the Spitzer image. While the $\mathrm{NH}_{3}(1,1)$ emission is spatially extended, the $\mathrm{NH}_{3}(2,2)$ emission is compact (Figure 2, top right), suggesting that the extended emission is at lower temperatures. We identified the most prominent filaments based on the morphology of the $\mathrm{NH}_{3}(1,1)$ together with the fact that these structures are coherent in velocity. We used the following criteria: (1) filaments must have aspect ratio larger than 6; (2) the signal-to-noise ratio should be larger than $9 ;{ }^{14}$ and (3) they must appear in at least two velocity channels and spanning a maximum velocity range of $3 \mathrm{~km} \mathrm{~s}^{-1}$. Figure 2 (top right) shows, for comparison, the $870 \mu \mathrm{m}$ continuum emission from the LABOCA bolometer at the APEX telescope (Busquet 2010), supporting our identification.

We identified a network of eight filaments and two hubs (named hub-N and hub-S in Figure 2), which were recognized using the $\mathrm{NH}_{3}(2,2)$ emission as denser regions in which some filaments intersect. The $\mathrm{NH}_{3}$ filaments, which cover a total area of $4.7 \times 8.7 \mathrm{pc}$, appear approximately parallel, in projection, in two preferred directions, at P.A. of $10^{\circ}$ and $60^{\circ}$, and they contain chains of dense cores ${ }^{15}$ aligned along the filament axis and distributed at somewhat regular spacings of about $\sim 30^{\prime \prime}$ or $0.33 \pm 0.09 \mathrm{pc}$ at the distance of the cloud. The averaged projected separation between adjacent filaments is between $0.5 \mathrm{pc}$ and $1 \mathrm{pc}$. In Table 1 we report on the length and width at FWHM of each filament obtained from $\mathrm{NH}_{3}(1,1)$ data. On

\footnotetext{
13 http://www.iram.fr/IRAMFR/GILDAS

14 Signal-to-noise ratio computed in the zero-order moment map of $\mathrm{NH}_{3}(1,1)$, where the rms noise level has been estimated using $3 \sigma \Delta v / \sqrt{3}$, where $\sigma$ is the rms noise of the channel maps and $\Delta v=0.6 \mathrm{~km} \mathrm{~s}^{-1}$.

15 Cores have been identified in the zero-order moment map of $\mathrm{NH}_{3}(1,1)$ if at least the $6 \sigma$ level is closed, where $\sigma$ is the rms noise of the map.
} 

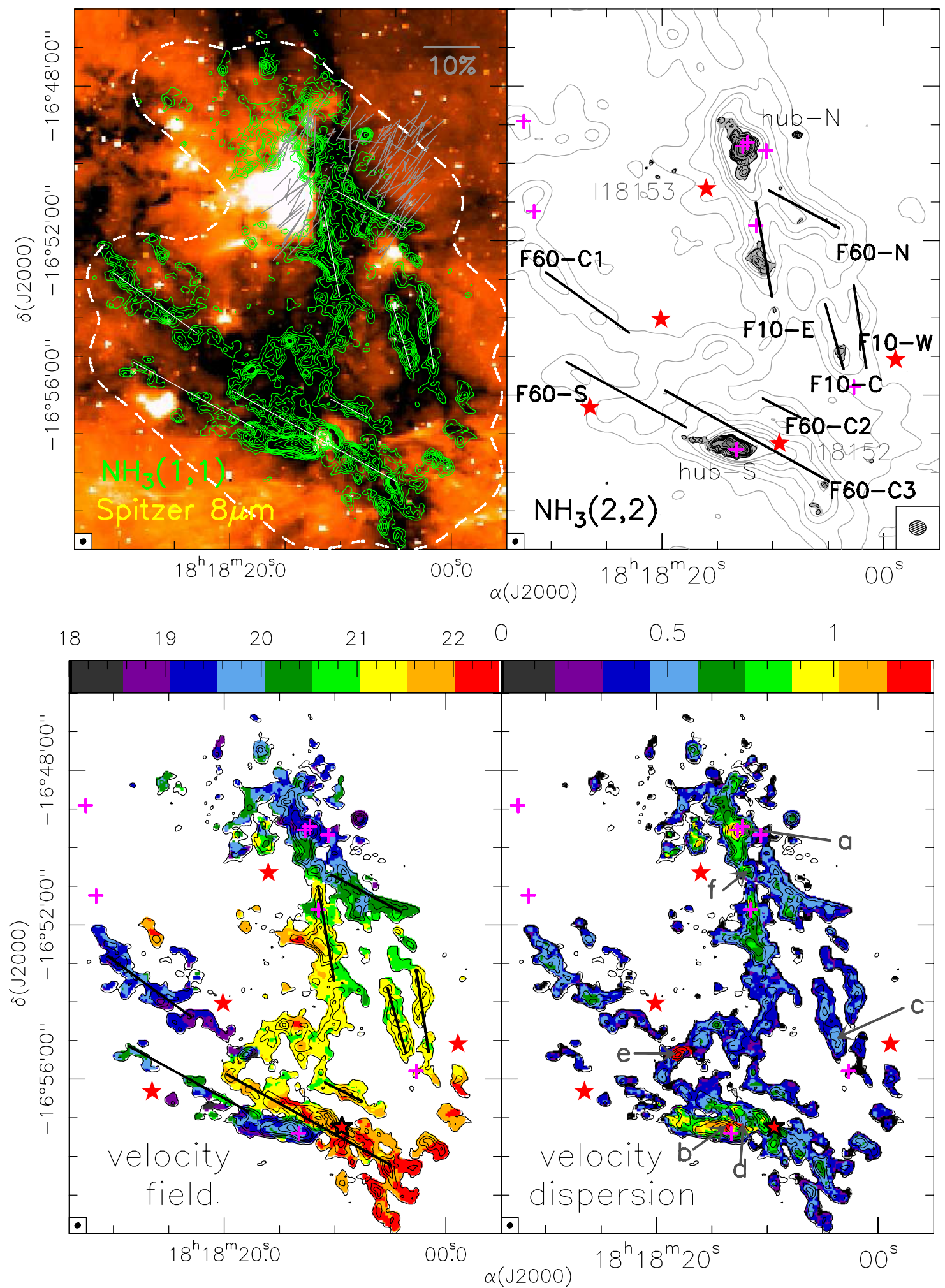

Figure 2. Top left: combined $\mathrm{NH}_{3}(1,1)$ integrated intensity (contours) overlaid on the $8 \mu \mathrm{m}$ Spitzer image (color scale). The contour levels range from 3 to 18 in steps of 3, and from 18 to 58 in steps of 10 times the rms noise of the map, 9 mJy beam ${ }^{-1} \mathrm{~km} \mathrm{~s}^{-1}$. The dashed line indicates $50 \%$ of the sensitivity level of the VLA mosaic. The gray lines are the polarization vectors of the near-infrared ( $H$-band) observations (G. Busquet et al., in preparation) with the scale shown in the top right corner. Top right: combined $\mathrm{NH}_{3}(2,2)$ integrated intensity (gray scale and black contours) overlaid on the $870 \mu$ m continuum from LABOCA bolometer at the APEX telescope (gray contours; Busquet 2010). The contour levels for $\mathrm{NH}_{3}(2,2)$ range from 2 to 10 in steps of 2, and from 10 to 60 in steps of 5 times the rms noise of the map, $9 \mathrm{mJy}_{\text {beam }}{ }^{-1} \mathrm{~km} \mathrm{~s}^{-1}$. The contour levels for $870 \mu \mathrm{m}$ emission range from 3 to 53 in steps of 10 , and from 53 to 653 in steps of 100 times the rms of the map, 25 mJy beam ${ }^{-1}$. The $\mathrm{NH}_{3}$ and $870 \mu \mathrm{m}$ continuum synthesized beams are shown in the bottom left and bottom right corners, respectively. Bottom: contours: zero-order moment map of $\mathrm{NH}_{3}(1,1)$. Color scale: $\mathrm{NH}_{3}(1,1)$ first-order moment map (left) and second-order moment map (right). Units are km s $\mathrm{s}^{-1}$. Stars indicate IRAS sources in the field, and crosses mark the position of $\mathrm{H}_{2} \mathrm{O}$ maser (Wang et al. 2006). The most prominent filaments are indicated with white/black lines and labeled according to its position angle. Arrows in the bottom right panel mark the positions of the $\mathrm{NH}_{3}(1,1)$ spectra shown in Figure 3 .

(A color version of this figure is available in the online journal.) 


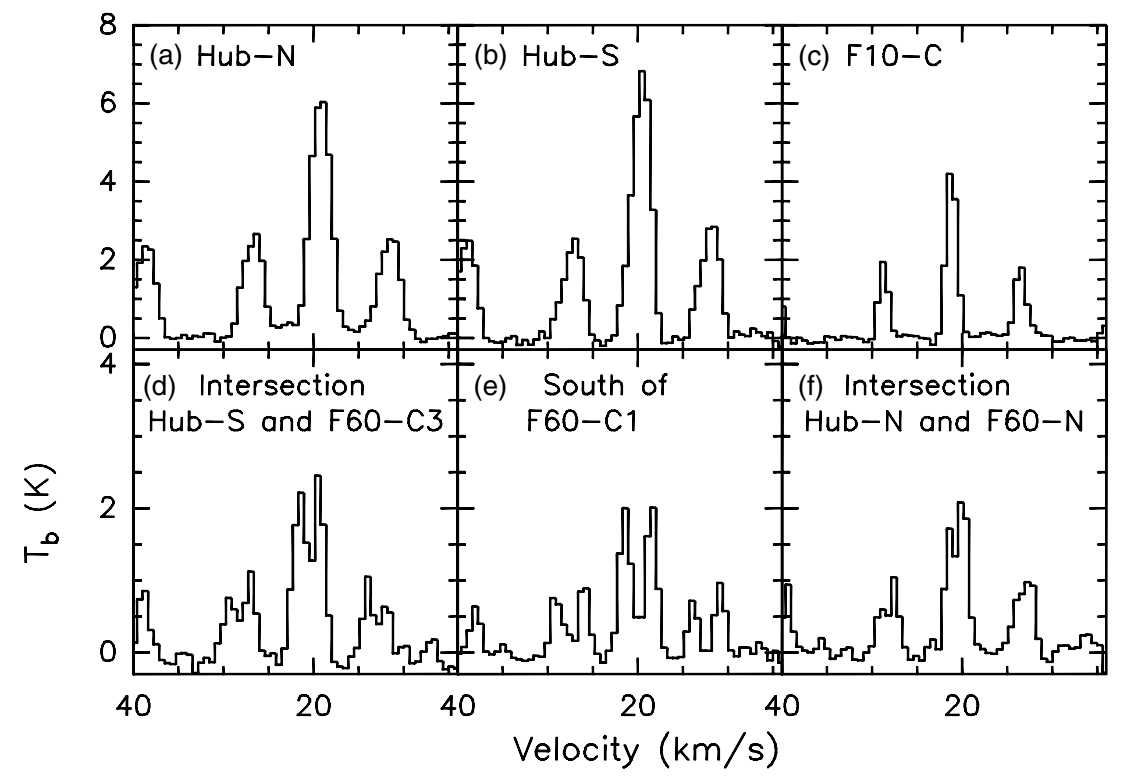

Figure 3. $\mathrm{NH}_{3}(1,1)$ spectra, in units of brightness temperature, at some selected positions depicted with arrows in Figure 2.

Table 1

Physical Properties of Filaments and Hubs

\begin{tabular}{|c|c|c|c|c|c|c|c|c|c|c|c|c|c|}
\hline Filament & $\begin{array}{l}\text { Length }^{\mathrm{a}} \\
(\mathrm{pc})\end{array}$ & $\begin{array}{l}\text { Width }^{\mathrm{a}} \\
(\mathrm{pc})\end{array}$ & $\begin{array}{c}\text { Aspect } \\
\text { Ratio }\end{array}$ & $\begin{array}{c}v_{\mathrm{LSR}} \\
\left(\mathrm{km} \mathrm{s}^{-1}\right)\end{array}$ & $\begin{array}{c}T_{\text {rot }}^{\mathrm{b}, \mathrm{c}} \\
(\mathrm{K})\end{array}$ & $\begin{array}{c}\sigma_{\mathrm{TOT}^{\mathrm{c}}} \\
\left(\mathrm{km} \mathrm{s}^{-1}\right)\end{array}$ & $\sigma_{\mathrm{NT}} / c_{\mathrm{s}}{ }^{\mathrm{c}}$ & $\begin{array}{c}M / l^{\mathrm{c}, \mathrm{d}} \\
\left(M_{\odot} \mathrm{pc}^{-1}\right)\end{array}$ & $\begin{array}{c}M_{\mathrm{vir}} / l^{\mathrm{l}, \mathrm{e}} \\
\left(M_{\odot} \mathrm{pc}^{-1}\right)\end{array}$ & $\alpha_{\mathrm{vir}}{ }^{\mathrm{e}}$ & $\begin{array}{c}\lambda_{\text {obs }}{ }^{f} \\
(\mathrm{pc})\end{array}$ & $\begin{array}{l}\lambda_{\mathrm{f}}{ }^{\mathrm{f}} \\
(\mathrm{pc})\end{array}$ & $N_{\text {cores }^{\mathrm{g}}}^{\mathrm{g}}$ \\
\hline F10-W & 1.48 & 0.11 & 13.5 & $20.8-22.0$ & $10 \pm 2$ & 0.49 & 2.38 & 105 & 112 & 1.1 & $0.27 \pm 0.08$ & $0.16-0.40$ & 6 \\
\hline F10-C & 1.18 & 0.14 & 8.4 & $20.8-22.0$ & $10 \pm 1$ & 0.50 & 2.31 & 147 & 115 & 0.8 & $0.33 \pm 0.07$ & $0.16-0.41$ & 4 \\
\hline F10-E & 1.63 & 0.15 & 10.9 & $20.2-22.0$ & $16 \pm 1$ & 0.98 & 3.63 & 87 & 448 & 5.1 & $0.32 \pm 0.11$ & $0.21-0.80$ & 10 \\
\hline F60-N & 1.23 & 0.09 & 13.7 & $19.5-20.8$ & $12 \pm 2$ & 0.54 & 2.31 & 149 & 135 & 0.9 & $0.25 \pm 0.09$ & $0.18-0.44$ & 7 \\
\hline F60-C1 & 1.81 & 0.12 & 15.1 & $18.3-20.2$ & $10 \pm 3$ & 0.63 & 3.10 & 120 & 185 & 1.5 & $0.38 \pm 0.11$ & $0.16-0.52$ & 8 \\
\hline F60-C2 & 0.66 & 0.09 & 7.3 & $20.8-22.6$ & $10 \pm 3$ & 0.61 & 3.03 & 74 & 174 & 2.3 & $0.49 \pm 0.09$ & $0.16-0.50$ & 5 \\
\hline F60-C3 & 3.34 & 0.12 & 27.8 & $21.4-23.2$ & $10 \pm 1$ & 0.73 & 3.67 & 218 & 248 & 1.1 & $0.25 \pm 0.12$ & $0.16-0.60$ & 14 \\
\hline F60-S & 2.12 & 0.10 & 21.2 & $18.3-20.8$ & $10 \pm 3$ & 0.98 & 5.02 & 132 & 447 & 3.4 & $0.38 \pm 0.11$ & $0.16-0.80$ & 6 \\
\hline Hub-N & 1.12 & 0.23 & 4.9 & $17.7-20.8$ & $15 \pm 1$ & 0.98 & 3.80 & 266 & 446 & 1.7 & $\ldots$ & $\ldots$ & \\
\hline Hub-S & 1.15 & 0.23 & 5.0 & $18.3-20.8$ & $15 \pm 1$ & 1.09 & 4.31 & 328 & 548 & 1.7 & $\cdots$ & $\cdots$ & \\
\hline
\end{tabular}

Notes.

${ }^{a}$ Deconvolved size at FWHM not corrected for projection effects.

${ }^{\mathrm{b}} T_{\text {rot }}$ has been derived following the appendix of Busquet et al. (2009).

${ }^{c}$ Averaged values within the area at FWHM.

d Mass per unit length, where the mass, $M=N\left(\mathrm{H}_{2}\right) 2.8 m_{\mathrm{p}} A$, has been estimated assuming an $\mathrm{NH}_{3}$ abundance of $3 \times 10^{-8}$ (average value measured in IRDCs; Pillai et al. 2006) and using the area $A$ of the filament at FWHM. The uncertainty in the mass is a factor of three.

e Virial mass per unit length $M_{\mathrm{vir}} / l=2 \sigma_{\mathrm{TOT}}^{2} / G$, and virial parameter $\alpha_{\mathrm{vir}}=M_{\mathrm{vir}} / M$ (Bertoldi \& McKee 1992).

${ }^{\mathrm{f}} \lambda_{\text {obs }}$ : observed separation between cores within a filament. $\lambda_{\mathrm{f}}$ : predicted core separation $\lambda_{\mathrm{f}}=22 H$, where $H=c_{\mathrm{s}}\left(4 \pi G 2.8 m_{\mathrm{H}} n_{\mathrm{c}}\right)^{-1 / 2}$ is the scale height, with $c_{\mathrm{s}}$ the isothermal sound speed (estimated by converting $T_{\text {rot }}$ to kinetic temperature using the expression of Tafalla et al. 2004), $G$ the gravitational constant, and $n_{\mathrm{c}}$ the gas density at the center of the filament, adopted to be $10^{5} \mathrm{~cm}^{-3}$. The first value corresponds to the core separation using $c_{\mathrm{s}}$ and the second value was obtained replacing $c_{\mathrm{s}}$ by $\sigma_{\mathrm{TOT}}$.

${ }^{g}$ Number of cores within each filament.

average, we found that the aspect ratio is $\sim 15: 1$, with a typical FWHM width of $\sim 0.12 \mathrm{pc}$. This value is close to the filament width of $0.1 \mathrm{pc}$ reported for the IC 5146, Aquila, and Polaris molecular clouds from Herschel observations (Arzoumanian et al. 2011).

In Figure 2 (bottom left) we present the first-order moment map of the $\mathrm{NH}_{3}(1,1)$ main line. Within each filament the velocity variations are small, in the range of $1-2 \mathrm{~km} \mathrm{~s}^{-1}$ (see Table 1), similar to other filamentary IRDCs (e.g., Jackson et al. 2010). This network of filaments seems to be separated into two main velocity components, one at $v_{\text {LSR }} \sim 18.3-20.8 \mathrm{~km} \mathrm{~s}^{-1}$ and another one at $v_{\text {LSR }} \sim 20.8-23.2 \mathrm{~km} \mathrm{~s}^{-1}$, which overlap in the hubs. The second-order moment map is presented in Figure 2 (bottom right), and shows that the velocity dispersion is locally enhanced $\left(\sigma \sim 1 \mathrm{~km} \mathrm{~s}^{-1}\right)$ toward hubs. Additionally, a high velocity dispersion $\left(\sigma \sim 1.6 \mathrm{~km} \mathrm{~s}^{-1}\right)$ is seen toward an arcshaped structure connecting filament F10-E with the southern filaments, in a small region intersecting filament F60-C1 and labeled as position "e" in Figure 2. In this region the large values of the velocity dispersion are due to the presence of two velocity components separated by $\sim 3 \mathrm{~km} \mathrm{~s}^{-1}$ (see Figure 3(e)). The presence of two velocity components is also found in regions where filaments intersect hubs (see Figures 3(d) and (f)). In contrast, all the other filaments appear more quiescent, with a typical velocity dispersion of $\sim 0.4-0.6 \mathrm{~km} \mathrm{~s}^{-1}$.

To obtain the main physical properties (rotational temperature $T_{\text {rot }}$, total velocity dispersion $\sigma_{\mathrm{TOT}}$, and mass per unit length $M / l$ ) of each filament, we extracted an averaged spectrum of $\mathrm{NH}_{3}(1,1)$ and $(2,2)$ over the filament area at FWHM. The results are reported in Table 1 . The rotational temperature ranges 
between $10 \mathrm{~K}$ and $16 \mathrm{~K}$. The total velocity dispersion of the gas ranges from $\sim 0.5 \mathrm{~km} \mathrm{~s}^{-1}$ up to $1.1 \mathrm{~km} \mathrm{~s}^{-1}$, and the nonthermal velocity dispersion over the isothermal sound speed, $\sigma_{\mathrm{NT}} / c_{\mathrm{s}}$, ranges between 2 and 5 , implying that filaments in G14.2 are characterized by supersonic non-thermal motions. In Table 1 we also list the mass and virial mass per unit length, the observed separation between cores, and the number of cores in each filament. Finally, the total surface density estimated by taking the spectrum averaged over all $\mathrm{NH}_{3}$ filaments is $\Sigma \simeq 1.9 \mathrm{~g} \mathrm{~cm}^{-2}$.

\section{DISCUSSION AND CONCLUSIONS}

In the previous section we presented the main properties of the two hub-filament systems in G14.225-0.506. The hubs are more compact (aspect ratio 5 versus 15$)$, warmer $\left(T_{\text {rot }} \simeq 15 \mathrm{~K}\right.$ versus $11 \mathrm{~K}$ ), and show larger velocity dispersion and larger masses per unit length than filaments. Interestingly, the hubs are associated with $\mathrm{H}_{2} \mathrm{O}$ maser emission (Wang et al. 2006) and mid-infrared sources (see Figures 1 and 2), and they are the main sites of stellar activity within the cloud.

The stability of the filaments can be studied by estimating the virial parameter $\alpha_{\mathrm{vir}}=M_{\mathrm{vir}} / M$ (Bertoldi \& McKee 1992), which is $\lesssim 2$ for all the filaments and hubs except for F10-E, and five out of eight filaments are near virial equilibrium $\left(\alpha_{\text {vir }} \simeq 1\right)$. This indicates that most of the filaments are unstable (collapsing) and probably undergoing fragmentation, compatible with the clumpy structure of G14.2. It is worth noting that filament F10$\mathrm{E}$ has $T_{\text {rot }}$ and velocity dispersion values similar to the hub properties. Filament F10-E presents some striations converging toward it. However, while hub-N and hub-S seem to be places where two different velocity structures converge, F10-E shows only one velocity component. We speculate that I18153, an UC H II region with $L \sim 10^{4} L_{\odot}$ (A. Sánchez-Monge 2012, private communication), may compress the gas, heating and injecting turbulence to this filament $\left(\alpha_{\text {vir }} \simeq 5\right)$. The interaction of this UC H II region with the dense gas is also seen in hub$\mathrm{N}$, where the $\mathrm{NH}_{3}(2,2) / \mathrm{NH}_{3}(1,1)$ map shows a local heating (Figure 4). The position-velocity (PV) plot along this hub (see Figure 4) reveals an inverted C-like structure, consistent with an expanding shell (Arce et al. 2011).

We investigated the fragmentation of filaments in the magnetohydrodynamic "sausage"-type instability scenario (Chandrasekhar \& Fermi 1953; see also Jackson et al. 2010), which predicts periodic separation between fragments (or cores) for a given density and isothermal sound speed. For an isothermal gas cylinder of finite radius $R$, the core separation can be expressed as $\lambda_{\mathrm{f}}=22 H$ for $R \gg H$, where $H$ is the scale height (see Table 1 for the formal expression). This is the case of G14.2, since $R$ and $H$ are 0.12 pc and 0.04 pc, respectively. Adopting a density of $10^{5} \mathrm{~cm}^{-3}$, we estimated the predicted core separation using first the isothermal sound speed, yielding $\lambda_{\mathrm{f}} \sim 0.16-0.21 \mathrm{pc}$, and then replacing $c_{\mathrm{s}}$ by the total velocity dispersion $\sigma_{\text {TOT }}$, which gives $\lambda_{\mathrm{f}} \sim 0.4-0.8 \mathrm{pc}$ (see Table 1 ). The observed separation, $\sim 0.33 \mathrm{pc}$, is in agreement with these two extreme cases. It is noteworthy that most of the cores appear to be elongated along the direction of the filament, which could imply the possibility of further fragmentation at smaller scales as observed in the IRDC G28.34+0.06-P1 (Zhang et al. 2009; Wang et al. 2011).

One of the most intriguing features of G14.2 is the network of filaments that are aligned in parallel. The filaments appear to take two preferred directions, one group at a P.A. of $10^{\circ}$, and the others at a P.A. of $60^{\circ}$. This network of filaments may
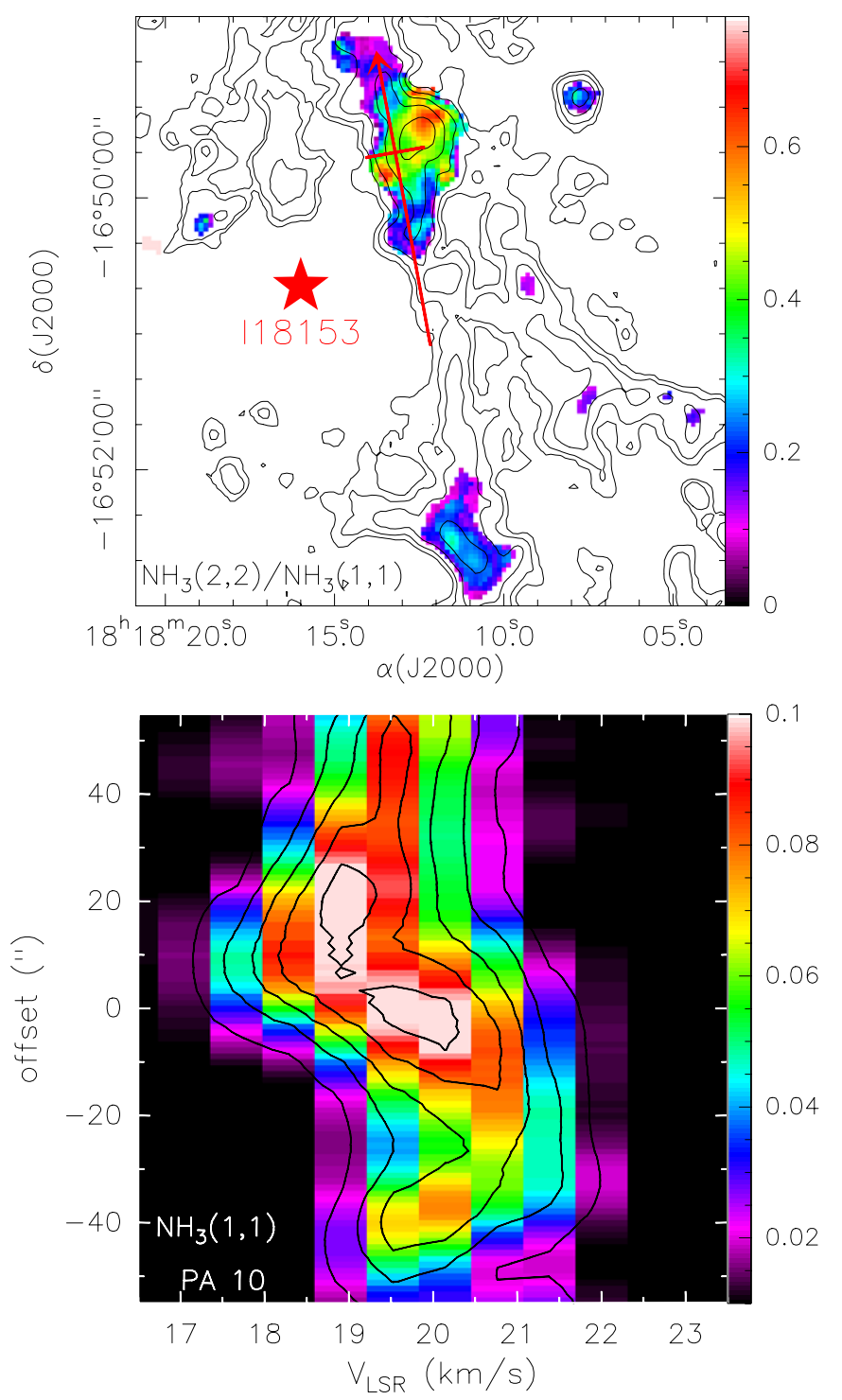

Figure 4. Top: $\mathrm{NH}_{3}(2,2) / \mathrm{NH}_{3}(1,1)$ map around I18153 (color scale) overlaid on the $\mathrm{NH}_{3}(1,1)$ integrated intensity (contours). The long red arrow depicts the position-velocity (PV) cut, where the center of the cut is indicated by the intersection of the two red lines. Bottom: PV plot of the $\mathrm{NH}_{3}(1,1)$ emission along the cut at P.A. $=10^{\circ}$. The contours start at 0.02 and increase in steps of $0.02 \mathrm{Jy} \mathrm{beam}^{-1}$. The positive offsets increase as indicated by the arrow.

(A color version of this figure is available in the online journal.)

arise from a layer of self-gravitating gas. Instability analysis has been performed for a layer of an isothermal infinite sheet under dynamical perturbation (Ledoux 1951; Schmid-Burgk 1967; Elmegreen \& Elmegreen 1978; Larson 1985; Nagai et al. 1998; Myers 2009). The gas is unstable to perturbations, which leads to high-density columns in the plane as a result of gravitational instability. The spacings between the high-density columns of gas correspond to the wavelength of the fastest growth mode. In the absence of magnetic fields, the growth of instability does not have a preferred direction in the plane. As a result, a grid of connected filaments appears in the gas layer. If the gas layer is threaded by magnetic fields, the growth of the instability develops unrestricted in one direction and is suppressed along the orthogonal direction. Nagai et al. (1998) analyzed a pressureconfined isothermal gas layer threaded by uniform magnetic fields. They found that in the regime of smaller external pressure (i.e., the scale height $H \ll Z_{\mathrm{b}}$, where $Z_{\mathrm{b}}$ is the thickness of 
the gas layer) the instability grows faster along the field lines. As a consequence, high-density columns or filaments develop with their longitudinal axis perpendicular to the field lines. In the high-pressure regime (i.e., $H \gg Z_{\mathrm{b}}$ ), the fastest growth of instability is perpendicular to the field lines and gives rise to filaments parallel to the magnetic fields.

Recent numerical simulations (S. Van Loo 2012, private communication) confirm the linear analysis in Nagai et al. (1998). The simulations show that an array of high-density columns develop in the gas layer with magnetic fields. In addition, lower density filamentary structures are also present, interconnecting the main filaments. The highest density structures are found at the intersections of major and minor filaments, as in this work. Furthermore, the simulations show that gravitational instability develops within a filament during the filament formation. This means that fragmentation of a filament into cores occurs simultaneously with the fragmentation of the sheet, but according to Toalá et al. (2012) with different free-fall times.

The array of filamentary structures in G14.225-0.506 may arise from gravitational instability of a thin gas layer with magnetic fields. In fact, preliminary near-infrared polarimetric observations around hub-N (G. Busquet et al. in preparation) reveal that the magnetic field is perpendicular to filaments at P.A. $\sim 60^{\circ}$ (see Figure 2). Therefore, according to Nagai et al. (1998), G14.2 would be in the regime of small external pressure $\left(H \ll Z_{\mathrm{b}}\right)$. Using the total surface density (see Section 3 ), the scale height $H$ of the initial gas layer is $H \sim 0.09 \mathrm{pc}$. This value should be regarded with caution, and to definitively assess its validity one would need observations of a low-density gas tracer to be sensitive to the gas layer. The wavelength of the fastest mode can be expressed as $\lambda_{\text {fastest }}=4 \pi H$ (Equation (60) in Nagai et al. 1998). Using our estimation of $H$, the predicted separation is $\sim 1.1 \mathrm{pc}$, in agreement with the observed filament separation (between 0.5 and $1 \mathrm{pc})$. It is not clear how such a large gas layer $(4.7 \times 8.7 \mathrm{pc})$ may form initially. The convergence of dynamic flows could be responsible for the formation of such a large gas layer that subsequently could fragment into parallel filaments as a result of magnetic modulation. Our $\mathrm{NH}_{3}$ data, although showing two velocity components, do not reveal evidence of converging/interacting flows and further observations of lowvelocity shock tracers, like $\mathrm{SiO}$ or $\mathrm{CH}_{3} \mathrm{CN}$ (Jiménez-Serra et al. 2010; Csengeri et al. 2011), and a tracer of low-density material are required to definitely identify signatures of converging flows. Overall, our data suggest that magnetic fields might play an important role in the alignment of filaments, and polarization measurements in the entire cloud would lend further support to this scenario.

The authors are grateful to the anonymous referee for valuable comments. G.B. is deeply grateful to Eugenio Schisano for very fruitful discussion on filaments. G.B. is funded by an Italian Space Agency (ASI) fellowship under contract number I/005/07/0. A.P., R.E., and I.d.G.-M. are supported by the Spanish MICINN grant AYA2011-30228-C03 (co-funded with
FEDER funds). A.P. is supported by a JAE-Doc CSIC fellowship co-funded with the European Social Fund, under the program "Junta para la Ampliación de Estudios," and by the AGAUR grant 2009SGR1172 (Catalonia). F.P.S. and G.A.P.F. are partially supported by CNPq and FAPEMIG. This work is partially based on observations with the $100 \mathrm{~m}$ telescope of the MPIfR (Max-Planck-Institut für Radioastronomie) at Effelsberg.

\section{REFERENCES}

André, P., Men'shchikov, A., Bontemps, S., et al. 2010, A\&A, 518, L102 Anglada, G., Estalella, R., Pastor, J., Rodríguez, L. F., \& Haschick, A. D. 1996, ApJ, 463, 205

Arce, H. G., Borkin, M. A., Goodman, A. A., Pineda, J. E., \& Beaumont, C. N. 2011, ApJ, 742, 105

Arzoumanian, D., André, P., Didelon, P., et al. 2011, A\&A, 529, L6

Bertoldi, F., \& McKee, C. F. 1992, ApJ, 395, 140

Bronfman, L., Nyman, L. -A., \& May, J. 1996, A\&AS, 115, 81

Busquet, G. 2010, PhD thesis, Univ. Barcelona

Busquet, G., Palau, A., Estalella, R., et al. 2009, A\&A, 506, 1183

Chandrasekhar, S., \& Fermi, E. 1953, ApJ, 118, 116

Csengeri, T., Bontemps, S., Schneider, N., et al. 2011, ApJL, 740, L5

Elmegreen, B., \& Elmegreen, D. 1978, ApJ, 220, 1051

Goldsmith, P. F., Heyer, M., Narayanan, G., et al. 2008, ApJ, 680, 428

Hacar, A., \& Tafalla, M. 2011, A\&A, 533, A34

Hatchell, J., Richer, J. S., Fuller, G. A., et al. 2005, A\&A, 440, 151

Heitsch, F., Hartmann, L. W., Slyz, A. D., Devriendt, J. E. G., \& Burkert, A. 2008, ApJ, 674, 316

Henshaw, J. D., Caselli, P., Fontani, F., et al. 2013, MNRAS, 428, 3425

Jackson, J. M., Finn, S. C., Chambers, E. T., Rathborne, J. M., \& Simon, R. 2010, ApJL, 718, L185

Jaffe, D. T., Güsten, R., \& Downes, D. 1981, ApJ, 250, 621

Jaffe, D. T., Stier, M. T., \& Fazio, G. G. 1982, ApJ, 252, 601

Jiménez-Serra, I., Caselli, P., Tan, J. C., et al. 2010, MNRAS, 406, 187

Larson, R. 1985, MNRAS, 214, 379

Ledoux, P. 1951, AnAp, 14, 438

Liu, H. B., Jiménez-Serra, I., Ho, P. T. P., et al. 2012, ApJ, 756, 10

Miettinen, O. 2012, A\&A, 542, A101

Molinari, S., Swinyard, B., Bally, J., et al. 2010, A\&A, 518, L100

Myers, P. C. 2009, ApJ, 700, 1609

Nagai, T., Inutsuka, S.-I., \& Miyama, S. M. 1998, ApJ, 506, 306

Nakamura, F., \& Li, Z.-Y. 2008, ApJ, 687, 354

Nakamura, F., Miura, T., Kitamura, Y., et al. 2012, ApJ, 746, 25

Palagi, F., Cesaroni, R., Comoretto, G., Felli, M., \& Natale, V. 1993, A\&AS, 101,153

Peretto, N., \& Fuller, G. A. 2009, A\&A, 505, 405

Pillai, T., Wyrowski, F., Carey, S. J., \& Menten, K. M. 2006, A\&A, 450, 569

Pineda, J. E., Goodman, A. A., Arce, H. G., et al. 2011, ApJL, 739, L2

Plume, R., Jaffe, D. T., \& Evans, N. J., II 1992, ApJS, 78, 505

Rathborne, J. M., Jackson, J. M., \& Simon, R. 2006, ApJ, 641, 389

Schmid-Burgk, J. 1967, ApJ, 149, 727

Schneider, N., Csengeri, T., Bontemps, S., et al. 2010, A\&A, 520, A49

Schneider, S., \& Elmegreen, B. G. 1979, ApJS, 41, 87

Tafalla, M., Myers, P. C., Caselli, P., \& Walmsley, C. M. 2004, A\&A, 416,191

Toalá, J. A., Vázquez-Semadeni, E., \& Gómez, G. C. 2012, ApJ, 744, 190

Vogel, S. N., Wright, M. C. H., Plambeck, R. L., \& Welch, W. J. 1984, ApJ, 283, 655

Wang, Y., Zhang, Q., Pillai, T., Wyrowski, F., \& Wu, Y. 2008, ApJL, 672, L33

Wang, Y., Zhang, Q., Rathborne, J. M., Jackson, J., \& Wu, Y. 2006, ApJL, 651, L125

Wang, K., Zhang, Q., Wu, Y., \& Zhang, H. 2011, ApJ, 735, 64

Wiseman, J. J., \& Ho, P. T. P. 1998, ApJ, 502, 676

Zhang, Q., Wang, Y., Pillai, T., \& Rathborne, J. 2009, ApJ, 696, 268 